\title{
A experiência do município de Paraty (Rio de Janeiro, Brasil) na prevenção e controle da leishmaniose tegumentar americana
}

\author{
CAROLINA M. DA COSTA*, FLÁVIO F. B. MOUTINHO** e SÁVIO F. BRUNO***
}

\section{THE PARATY COUNTY (RIO DE JANEIRO, BRAZIL) EXPERIENCE IN AMERICAN TEGUMENTARY LEISHMANIOSIS PREVENTION AND CONTROL}

American tegumentary leishmaniosis (ATL) is an important zoonosis in Rio de Janeiro State, Brazil, and it represents an increasing public health problem. The municipality of Paraty is an endemic area, where 76 human cases were reported in 2002. In June of 2002 the Municipal Health Department had started an ATL control program, according with methodologies recommended by National Health Foundation (FUNASA) on the ATL Control Manual of 2000. The control strategies were based on capture of sandflies in Atlantic Forest areas and identification of vectors species, chemical control of vectors through periodic domiciliary and peridomiciliary insecticide application, serological investigation of suspect dogs and elimination of infected dogs, rodents control, investigation of new possible cases and educational measures to the local community. This paper presents the municipality of Paraty experience on implanting a control program of ATL and its efficiency. After the adoption of these measures, it had occurred 52 new cases in 2003. This result indicates that there was a decrease of 31,6\%, in relation to the cases reported on 2002.

Key words: American tegumentary leishmaniaosis, Rio de Janeiro State-Brazil, control measures.

\section{INTRODUÇÃO}

A leishmaniose tegumentar americana (LTA), zoonose que se distribui amplamente no continente americano, é considerada pela Organização Mundial de Saúde como uma das seis mais importantes doenças infecciosas, pela sua alta incidência e potencial capacidade de produzir deformações ${ }^{1}$. Representa um importante problema de saúde pública, com manifestações clínicas que variam desde lesões cutâneas simples a mucocutâneas e lesões disseminadas².

No Brasil, essa doença apresenta-se em franco crescimento, tanto em magnitude, como em expansão geográfica. No período de 1970 a 2001, o número de casos notificados de LTA, elevouse de 3.000 para $37.000^{3}$.

Atualmente considera-se a Leishmania

\footnotetext{
* Aluna de Mestrado do Curso de Pós Graduação em Ciências Veterinárias-Medicina Veterinária Coletiva da Universidade Federal Fluminense-UFF.

** Médico Veterinário-Diretor da Divisão de Vigilância Sanitária de Paraty - RJ.

****Prof. Orientador-Coordenador da Disciplina Medicina da Conservação do Curso de Pós Graduação em Ciências Veterinárias-UFF. Rua Vital Brazil Filho, 64. CEP 24.230-340. Niterói-Rio de Janeiro-Brasil.
} 
(Viannia) braziliensis o principal agente etiológico dessa enfermidade no Rio de Janeiro ${ }^{4-6}$.

A leishmaniose tegumentar americana é de ocorrência endêmica no Estado do Rio de Janeiro, sendo a maior incidência observada nos municípios do litoral sul ${ }^{7}$. Nesse Estado, o município de Paraty detém o segundo maior número de casos da doença ${ }^{8}$, registrando 76 novos casos no ano de 2002. As regiões mais acometidas estão situadas na encosta da Serra do Mar parcialmente recoberta pela Mata Atlântica, onde observa-se uma ocupação humana desordenada, associada ao desmatamento.

No intuito de reduzir o número de casos no município, a Secretaria de Saúde de Paraty iniciou, em junho de 2002, um programa de controle da LTA, de acordo com a metodologia proposta pela Fundação Nacional de Saúde (FUNASA) no Manual de controle da LTA de 2000. O presente trabalho objetiva abordar a situação atual da LTA neste município, avaliando de forma preliminar as causas correlatas à incidência desta enfermidade e descrevendo as ações de controle empregadas na atualidade.

\section{MATERIALE MÉTODO}

Área de trabalho: O Município de Paraty tem grande importância para o Estado, sendo o turismo, a pesca e a agricultura, os alicerces de sua economia?.

Localiza-se no extremo sul do Estado do Rio de Janeiro, ocupando uma área de $917 \mathrm{Km}^{2}$. Apresenta uma população residente estimada para 2003, segundo o censo 2000, de 31.275 habitantes, a maioria residente das áreas rurais ${ }^{10}$. O clima é quente úmido na base da serra e subtropical úmido nas regiões serranas. A fauna é rica em animais silvestres, à exemplo, capivaras, pacas, quatis ${ }^{7}$ e representantes das Ordens Xenarthra e Marsupialia.

$\mathrm{Na}$ seleção das áreas para a execução das atividades do programa de controle da LTA, considerou-se as que apresentavam a maior incidência de casos da doença em humanos e animais do município. Uma parte destas encontrase na faixa litorânea e outra parte em regiões de relevo, situadas na periferia do município, sendo todas consideradas áreas rurais.

Busca ativa de novos casos: Residências localizadas nas áreas costeiras e rurais do município foram visitadas por membros da equipe de Vigilância Sanitária responsável pelas ações de controle da LTA. Na abordagem de cada casa, os moradores foram entrevistados para a obtenção de informações como o nome, idade e residência. A equipe também investigou a presença de lesões sugestivas e/ou cicatrizes nas pessoas e animais.

Pesquisa entomológica: Foram realizadas capturas quinzenais de flebotomíneos em casas localizadas em áreas periurbanas e rurais do município, onde a incidência da enfermidade tem sido alta tanto em humanos quanto em animais. As capturas foram efetuadas em 2 sítios de coleta: no ambiente intradomiciliar, nas paredes internas, utilizando-se isca humana; e no ambiente peridomiciliar, nas paredes externas, árvores e anexos como galinheiros e chiqueiros. Para a coleta dos insetos utilizou-se tubo de sucção manual (capturador de Castro). As capturas foram realizadas no período das $18 \mathrm{~h}$ às $21 \mathrm{~h}$.

Os flebotomíneos capturados eram transferidos para tubos contendo álcool $70 \%$ e transportados para o laboratório entomológico da Secretaria Municipal de Saúde, para serem preparados e identificados.

Controle químico do vetor: Borrifações intra e peridomiciliares de inseticida foram realizadas periodicamente, com o auxílio de bombas de aspersão manual contendo piretróide e em alguns casos, carbamatos. Foram borrifadas as paredes internas, paredes externas e anexos dos domicílios visitados. Nas localidades mais acometidas pela LTA, realizou-se, também, aspersão espacial de inseticida (termonebulização) a cada 40 dias utilizando-se piretróide diluído em óleo mineral, objetivando maior efeito residual do inseticida.

Controle de possíveis reservatórios: Animais domésticos que apresentavam lesões suspeitas de LTA foram submetidos a testes sorológicos por imunofluorescência indireta. Os positivos foram sacrificados com a permissão dos proprietários.

Controle de roedores: Os roedores são animais sinantrópicos, reservatórios de leishmaniose tegumentar, com taxas elevadas de infecção ${ }^{11}$. Devido a isso, a atividade de controle dos roedores também fez parte do programa de controle da LTA realizado no município. O raticida empregado foi o Difethialone em forma de bloco parafinado e "pelet". Preenchia-se uma ficha de controle de roedores em cada casa visitada, identificando a característica do imóvel e dos achados que poderiam estar relacionados com a presença 
desses animais.

Educação em saúde: A Secretaria de Saúde do município de Paraty promoveu ações de educação ambiental e educação em saúde com a população local, através de palestras e oficinas. Estas também fazem parte do Programa de Controle da LTA, sendo realizadas periodicamente. As atividades de educação têm por objetivo levar às comunidades do município informações sobre a leishmaniose tegumentar americana, seu tratamento, suas causas e as formas de prevenção.

\section{RESULTADOS}

Desde o início do programa de controle da LTA, em junho de 2002 até junho de 2003, 3.585 imóveis foram borrifados com inseticida e desratizados. Nesse período, foram realizadas 36 capturas de flebotomíneos nas localidades mais acometidas do município. Foram capturados um total de 1.109 flebotomíneos (Tabela 1), sendo Lutzomyia intermedia a espécie predominante (85,8\%), seguido de Lu. fischeri $(7,1 \%)$.

Após a implantação do programa de controle da LTA, foram registrados 52 novos casos no ano de 2003, indicando uma queda de $31,6 \%$ em relação a 2002 (Figura 1).

\section{DISCUSSÃO}

As atividades de controle da leishmaniose tegumentar americana usualmente empregadas baseiam-se em medidas de atuação na cadeia de transmissão, através da redução de vetores, eliminação de reservatórios infectados, vigilância e tratamento de casos humanos, proteção pessoal e medidas educativas. Em virtude das caracte-

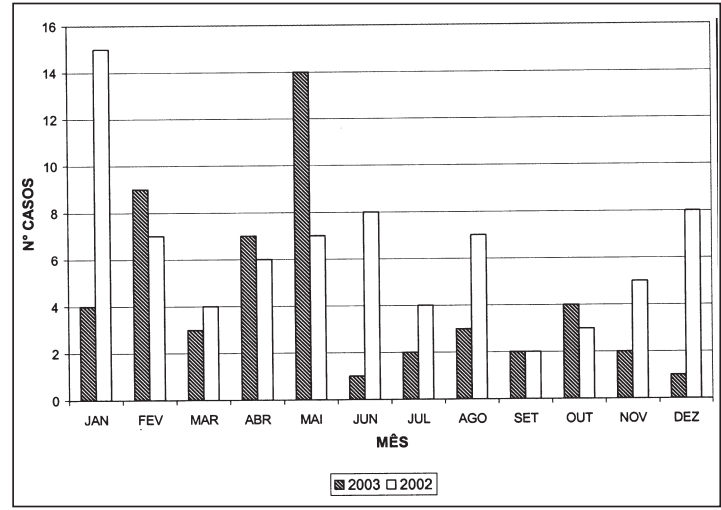

Figura 1. Número de casos humanos de LTA ocorridos em Paraty, estado do Rio de Janeiro, nos anos de 2002 e 2003.

rísticas epidemiológicas peculiares da LTA, as estratégias de controle devem ser flexíveis e distintas, adequadas a cada região ou foco particular ${ }^{11}$.

Conforme observado, as regiões do município em estudo situam-se em áreas de relevo e faixas litorâneas, ambos recobertos por remanescentes de Mata Atlântica. Muitas destas localidades apresentam-se em processo progressivo de ocupação humana, que vem ocorrendo de forma desordenada, associado a um crescente desmatamento e conseqüentes alterações ambientais.

Essas alterações, de origem antrópica, estão modificando o perfil epidemiológico da leishmaniose em áreas onde a $L$. braziliensis era mantida predominantemente em um ciclo silvestre. A adaptação do flebotomíneo ao ambiente peridomiciliar ou até mesmo domiciliar propicia a transmissão da Leishmania a animais domésticos como cães e eqüinos, assim como

Tabela 1. Quantidade de exemplares e distribuição das espécies de flebotomíneos de acordo com o ponto de captura e sexo, no período de junho de 2002 a junho de 2003 em Paraty, estado do Rio de Janeiro

\begin{tabular}{|c|c|c|c|c|c|c|c|c|c|}
\hline \multirow{3}{*}{$\begin{array}{l}\text { Ponto de } \\
\text { Captura }\end{array}$} & \multicolumn{8}{|c|}{ Espécies } & \multirow{3}{*}{ Total } \\
\hline & \multicolumn{2}{|c|}{ Lu. intermedia } & \multicolumn{2}{|c|}{ Lu. fischeri } & \multicolumn{2}{|c|}{ Lu. migonei } & \multicolumn{2}{|c|}{ Lu. edwardsi } & \\
\hline & $\mathbf{F}$ & M & $\mathbf{F}$ & M & $\mathbf{F}$ & M & $\mathbf{F}$ & $\mathbf{M}$ & \\
\hline Parede externa & 120 & 182 & 6 & 25 & 1 & 3 & 0 & 1 & 338 \\
\hline Parede interna & 53 & 117 & 4 & 9 & 3 & 5 & 0 & 0 & 191 \\
\hline Árvore & 246 & 60 & 5 & 14 & 15 & 27 & 0 & 0 & 367 \\
\hline Galinheiro & 80 & 48 & 2 & 10 & 8 & 15 & 0 & 0 & 163 \\
\hline Chiqueiro & 14 & 32 & 1 & 3 & 0 & 0 & 0 & 0 & 50 \\
\hline Total & 513 & 439 & 18 & 61 & 27 & 50 & 0 & 1 & 1.109 \\
\hline
\end{tabular}

$\mathrm{F}$ = fémea; $\mathrm{M}=$ macho. 
ao homem, tornando a probabilidade de transmissão semelhante em toda a população que se encontra sob risco, não importando a faixa etária, sexo ou atividade profissional ${ }^{12}$.

Analisando-se a fauna flebotomínica de Paraty verificou-se que, das quatro espécies capturadas, Lu. intermedia foi a espécie predominante, sendo freqüentemente encontrada dentro das habitações humanas e nos peridomicílios. Pesquisa realizada em 1991 no mesmo município, já havia incriminado $L u$. intermedia como a principal transmissora da doença na região, devido a sua prevalência e antropofilia. Embora o município esteja localizado junto a Floresta Atlântica, tem sido observado que a transmissão da leishmaniose vem ocorrendo no ambiente domiciliar e perido-miciliar, em especial, devido ao comportamento da $L u$. intermedia, com significante incidência em indivíduos que não freqüentam regularmente a floresta, incluindo crianças muito pequenas ${ }^{13}$.

O combate ao vetor e ao reservatório é uma medida que geralmente leva à diminuição da transmissão peridomiciliar ${ }^{12}$. Em contraposição, não é difícil pressupor que na experiência de Paraty, o combate aos reservatórios traduz-se não só a ações ecologicamente impactantes, como ilegais, considerando que os reservatórios (comprovados ou supostos) compõem fração da fauna de vertebrados silvestres deste país, como marsupiais, carnívoros, roedores e mesmo primatas não humanos.

Quanto aos animais domésticos, há necessidade de se realizar pesquisas para evidenciar o verdadeiro papel destes animais como possíveis reservatórios no ambiente peri e intradomiciliar. A Fundação Nacional de Saúde preconiza que cães e eqüinos positivos para leishmaniose tegumentar sejam mantidos em locais limpos e afastados de habitações humanas. $\mathrm{Na}$ prática, contudo, essas medidas são de complexa introdução.

Em locais onde o foco de transmissão é domiciliar e peridomiciliar, intervenções que diminuem a abundância de flebotomíneos e as taxas de picadas dentro e ao redor das casas pode reduzir a incidência da doença ${ }^{14}$. Deve-se considerar entretanto, que nesta região, as residências encontram-se muitas vezes literalmente dentro da mata. Este fato demostra a importância relativa das aplicações periódicas de inseticida nos domicílios localizados nas áreas mais acometidas. A redução do contato homem- vetor nestes casos provavelmente é pouco significante, principalmente quando são utilizados inseticidas de baixo efeito residual.

As atividades de educação em saúde provavelmente contribuíram para o controle da leishmaniose tegumentar americana, pois a população passou a colaborar mais com as equipes de Vigilância Sanitária, facilitando a realização das atividades de controle, assim como os pacientes portadores de lesão, passaram a procurar assistência médica mais rapidamente.

A importância da LTA reside não somente na alta incidência e ampla distribuição geográfica, mas também na possibilidade de assumir formas que podem determinar lesões destrutivas e desfigurantes, com grande repercussão no campo psicossocial do indivíduo ${ }^{15}$. Além disso, o aumento do número de casos desta enfermidade eleva os gastos governamentais. Ressalta-se que o medicamento utilizado para o tratamento (Glucantime) é oferecido gratuitamente pela Fundação Nacional de Saúde.

Enquanto uma vacina segura e eficaz não se encontra disponível, as formas de controle que utilizam métodos mais tradicionais (redução de vetores, eliminação de reservatórios infectados, proteção pessoal, vigilância e tratamento) traduzem-se na melhor opção ${ }^{16}$.

\section{RESUMO}

A leishmaniose tegumentar americana (LTA) é uma importante zoonose no Estado do Rio de Janeiro, Brasil, e representa um grande problema de saúde pública. O município de Paraty vem apresentando altas taxas desta doença, sendo registrados 76 casos no ano de 2002. Na tentativa de reduzir o número de casos no município, a Secretaria de Saúde de Paraty iniciou, em junho de 2002, um programa de controle da LTA, de acordo com a metodologia proposta pela Fundação Nacional de Saúde (FUNASA) no Manual de controle da LTA de 2000, adaptado às peculiaridades locais. O conjunto de medidas adotadas baseou-se na captura de flebotomíneos em áreas de Mata Atlântica e identificação das espécies vetoras, ações de controle químico do vetor através da aplicação periódica de inseticidas intra e peridomiciliares, inquérito sorológico dos cães suspeitos e eutanásia dos positivos, controle de roedores, busca ativa de possíveis novos casos e atividades de educação e saúde com a população 
residente. O presente trabalho tem como objetivo apresentar a experiência do município de Paraty, estado do Rio de Janeiro, Brasil, na implantação do programa de controle da LTA e sua eficácia, considerando-se que, após a adoção de tais medidas, foram registrados 52 novos casos no ano de 2003. Este resultado indica uma queda de $31,6 \%$, comparando-se com os dados obtidos em 2002.

\section{REFERÊNCIAS}

1.- MINISTÉRIO DA SAÚDE. Boletim epidemiológico. Fundação Nacional de Saúde. Ano III, edição especial. Brasília, 1999.

2.- MACHADO-COELHO G L L, ASSUNÇÃO R, MAYRINK W et al. American cutaneous leishmaniasis in southeast Brazil: space-time clustering. Int J Epidem 1999; 28: 982-9.

3.-- CENTRO NACIONAL DE EPIDEMIOLOGIACENEPI. Boletim Epidemiológico. Fundação Nacional de Saúde-Ministério da Saúde. Ano II, N. ${ }^{\circ}$ 5. Brasília, 2002.

4.- GRIMALDI Jr G, TESH R B, McMAHON-PRATT D. A review of geographic distribution and epidemiology of leishmaniasis in the New World. Amer. J Trop Med Hyg 1989; 41: 687-725.

5.- MARZOCHI M C A, MARZOCHI K B F. Tegumentary and visceral leishmaniasis in Brazil- emerging anthropozoonosis and possibilities for their control. Cadernos de Saúde Pública 1994; 10: 359-75.

6.- OLIVEIRA-NETO M P, MATTOS M S, PEREZ M A et al. American tegumentary leishmaniasis (ATL) in Rio de Janeiro state, Brazil: main clinical and epidemiologic characteristics. Int J Dermatol 2000; 9: 506.

7.- BARBOSA G M S, MARZOCHI M C A, MASSARD C L, CONFORT E M. Aspectos epidemiológicos da leishmaniose tegumentar americana em cães, no Município de Paraty, Estado do Rio de Janeiro, Brasil.
Cadernos de Saúde Pública 1999; 15: 641-6.

8.- GONCALVES R G, JORGE M E, SOUZA W J S et al. Estudo clínico da leishmaniose tegumentar no município de Paraty entre os anos de 1991 e 1997. XVIII Congresso Brasileiro de Parasitologia, Rio de JaneiroRJ. Livro de resumos, 2003.

9.- RODRIGUES A A. Paraty, Três Séculos de História. Paraty: Secretaria de Turismo e Cultura, Prefeitura Municipal de Paraty, 1995.

10.- INSTITUTO BRASILEIRO DE GEOGRAFIA E ESTATÍSTICA-IBGE. Censo demográfico 2000. Tabela- População residente por sexo e situação do domicílio, população residente de 10 anos ou mais de idade, total, alfabetizada e taxa de alfabetização, segundo os municípios. Disponível em: http:// www.ibge.gov.br. Acesso em 16 de abril de 2004, Brasil.

11.- MINISTÉRIO DA SAÚDE. Manual de controle da leishmaniose tegumentar americana. $5^{\mathrm{a}}$ ed. Brasília: Brasil. Fundação Nacional de Saúde, 2000. 62 p.

12.- DA-CRUZ A M, AZEREDO-COUTINHO K B. Leishmaniose Tegumentar Americana. In: Batista RS, Igreja RP, Gomes AD, Huggins DW. Medicina Tropical - Abordagem Atual das Doenças Infecciosas e Parasitárias. Rio de Janeiro: Ed. Cultura Médica, 2001.

13.- AGUIAR G M, MEDEIROS W M, DOS SANTOS T G et al. Ecology of sandflies in a recent focus of cutaneous leishmaniasis in Paraty, littoral of Rio de Janeiro state (Diptera, Psychodidae, Phlebotominae). Mem Inst Oswaldo Cruz 1993; 88: 339-340.

14.- CAMPBELL-LENDRUM D, DUJARDIN J P, MARTINEZ E et al. Domestic and peridomestic transmission of american cutaneous leishmaniasis: changing epidemiological patterns present new control opportunities. Mem Inst Oswaldo Cruz 2001; 6: 159 62.

15.- GONTIJO B, CARVALHO M L R. Leishmaniose tegumentar americana. Rev Soc Brasil Med Trop 2003; 36: $71-80$

16.- GRIMALDI Jr G, TESH R B. Leishmaniasis of the New World: Current concepts and implications for future research. Clin Microbiol Rev 1993; 6: 23050 . 